Márcio Augusto PINTO de Ávila ${ }^{1}$

LunNa Perdigão Borges²

Marina Silva Paez

Ricardo VASCONCELOS BRUNO ${ }^{4}$

ANTONIO EGÍDIO NARDI $\left.\right|^{5}$

Ana Carolina Machado de Pessôa ${ }^{6}$

Everyn de Souza PalmerRA ${ }^{6}$

Artigo Original

Palavras-chave

Acantose nigricans

Síndrome do ovário policístico

Resistência à insulina

Síndrome X metabólica

Obesidade

Keywords

Acanthosis nigricans Polycystic ovary syndrome Insulin resistance

Metabolic syndrome $X$

Obesity

\title{
Acantose nigricante: inter-relações metabólicas inerentes à síndrome dos ovários policísticos
}

\author{
Acanthosis nigricans: metabolic interrelations \\ inberent to the polycystic ovary syndrome
}

\section{Resumo}

OBJETIVO: Estabelecer a prevalência da acantose nigricante (AN) no contexto da síndrome dos ovários policísticos (SOP) e as respectivas associações com a obesidade, a resistência insulínica (RI), a insulinemia e a síndrome metabólica (SM). MÉTODOS: Em um estudo transversal e prospectivo, foram selecionadas cem pacientes acometidas pela SOP, diagnosticadas segundo o Consenso de Rotterdam (2003). O exame cutâneo incluiu, além da verificação da presença da AN, a presença do hirsutismo (escore $\geq 8$ ) e da acne. Foram investigados os dados clínicos e bioquímicos, os fatores de risco cardiovascular que se fazem presentes na SM, como circunferência abdominal (CA), obesidade, hipertensão e os índices de HDL e triglicérides. $\bigcirc$ modelo de aferição da resistência insulínica foi realizado por meio do teste homeostatic model assessment of insulin resistance (HOMA-IR). RESULTADOS: A prevalência da AN (53\%) mostrou correspondência significativa com o hirsutismo ( $p=0,02)$, o índice de massa corpórea (IMC) ( $p<0,01$ ), a insulinemia basal $(p<0,01)$, o HOMA-IR $(p<0,01)$ e a $S M(p<0,05)$. A SM alcançou a prevalência de 36\% e associou-se significativamente apenas com a $A N(p<0,01)$. Conquanto ausente o diabetes mellitus, sobressaem as conotações do HOMA-IR alterado $(p=0,01)$ com a $S M(p<5 \%)$ e a AN $(p<0,01)$. CONCLUSÕES: A AN integra o quadro fenotípico grave da SOP como mais um signo previsível dos riscos da doença cardiovascular.

Abstract

PURPOSE: To establish the prevalence of acanthosis nigricans (AN) within the context of polycystic ovary syndrome (PCOS) and its associations with obesity, insulin resistance, insulinemia, and metabolic syndrome (MS). METHODS: A cross-sectional prospective study was conducted on 100 patients with PCOS diagnosed according to the Rotterdam Consensus (2003). The skin test included, in addition to the detection of the presence of $A N$, the presence of hirsutism (score $\geq 8$ ) and acne. In addition to clinical and biochemical data, we investigated cardiovascular risk factors present in MS such as abdominal circumference, obesity, hypertension and HDL and triglyceride levels. Insulin resistance was determined by the HOMA-IR test. RESULTS: The prevalence of AN (53\%) was significantly correlated with hirsutism (p=0.02), body mass index $(p<0.01)$, basal insulinemia $(p<0.01)$, (HOMA-IR) $(p<0.01)$, and MS $(p<0.01)$. The prevalence of MS reached $36 \%$ and was significantly associated only with $A N(p<0.01)$. Although diabetes mellitus was absent, there was a significant association of altered HOMA-IR (p<0.01) with MS (p<5\%) and AN (p<0.01). CONCLUSION: AN is part of the severe phenotypic profile of PCOS as an additional predictable sign of the risks of cardiovascular disease.

Correspondêncic

Márcio Augusto Pinto de Ávilo Instituto de Ginecologia da Universidade Federal do Rio de Janeiro Rua Moncorvo Filho, 90 - Centro

CEP: 20211-340

Rio de Janeiro (RJ), Brasil

Recebido

$02 / 07 / 2014$

Aceito com modificacōos

$16 / 07 / 2014$
Instituto de Ginecologia, Instituto de Psiquiatria, Instituto de Matemática e Faculdade de Medicina, Universidade Federal do Rio de Janeiro - UFRJ - Rio de Janeiro (RJ), Brasil.

'Departamento de Ginecologia e Obstetrícia, Faculdade de Medicina, Universidade Federal do Rio de Janeiro - UFRJ - Rio de Janeiro (RJ), Brasil.

${ }^{2}$ Graduação em Estatística, Instituto de Matemática, Universidade Federal do Rio de Janeiro - UFRJ - Rio de Janeiro (RJ), Brasil. ${ }^{3}$ Departamento de Métodos Estatísticos, Instituto de Matemática, Universidade Federal do Rio de Janeiro - UFRJ - Rio de Janeiro (RJ), Brasil. ${ }^{4}$ Setor de Ginecologia Endócrina, Instituto de Ginecologia, Universidade Federal do Rio de Janeiro - UFRJ - Rio de Janeiro (RJ), Brasil. ${ }^{5}$ Departamento de Psiquiatria, Universidade Federal do Rio de Janeiro - UFRI - Rio de Janeiro (RI), Brasil.

${ }^{6}$ Curso de Graduação em Medicina, Universidade Federal do Rio de Janeiro - UFRJ - Rio de Janeiro (RJ), Brasil.

Conflito de interesses: não há 


\section{Introdução}

Considerados os aspectos diversos e a gradação das lesões cutâneas, tem-se, não de todo dirimidos, os mecanismos que assistem a intervenção da resistência insulínica (RI) na gênese de alterações que demandam o possível envolvimento de distúrbios metabólicos conexos à condição hormonal imperfeita, como sucede na vigência das "ligações perigosas" - hiperinsulinemia e hiperandrogenemia. $\mathrm{O}$ binômio hirsutismo-acne refere-se, em geral, às manifestações dérmicas comuns e esperadas na síndrome do ovário policístico (SOP), mas é necessário distinguir a inclusão da acantose nigricante (AN) entre essas alterações fenotípicas da SOP. Localizada, na maioria das vezes, na região cervical, o pescoço se vê acometido pela AN (de modo isolado ou combinado aos distintos segmentos) em 93 a $99 \%$ dos $\operatorname{casos}^{1}$, notadamente na porção posterior, ademais de diferentes áreas como face lateral do pescoço, axilas, tórax, superfície flexora dos membros, virilha, vulva e superfícies intertriginosas, conquanto raramente a planta dos pés e a palma das mãos ${ }^{2,3}$. O maior avanço no que concerne ao entendimento da $\mathrm{AN}$, não apenas submetida à indução pelo câncer, diz respeito ao vínculo com a $\mathrm{RI}^{4,5}$. Nesse ínterim, observações mais recentes dão conta da presença mais pronunciada da AN em circunstâncias peculiares, vale salientar: a obesidade, a diabete e a SOP. Pacientes com SOP e história familiar de diabete e obesidade, com índice de massa corpórea (IMC) superior a $30 \mathrm{~kg} / \mathrm{m}^{2}$ e circunferência abdominal (CA) $\geq 90 \mathrm{~cm}$ foram mais propensas a desenvolver AN na qualidade de marcador de hiperinsulinemia e $\mathrm{RI}^{6}$. Todavia, excetuada a SOP, a prevalência da AN demonstrou-se correlacionada à gravidade do peso corporal em $74 \%$ dos adultos obesos ${ }^{7}$. Em geral, tais indivíduos convivem com níveis plasmáticos de insulina em jejum elevados, comparativamente àqueles preservados da alteração cutânea. A propósito, estudos distinguem a hiperinsulinemia como uma condição de marcador da $\mathrm{AN}^{7}$, em vez de atribuir maior importância à adequação estrita com a obesidade.

Os fundamentos de cunho metabólico e endócrino implícitos a AN se inter-relacionam com a heterogeneidade fenotípica inerente à SOP, determinante, em princípio, da notável diversificação de relações mórbidas que possivelmente envolvem diferenciadas perspectivas de acometimento cutâneo. Por outro lado, o conceito que aglutina a constelação de fatores presumíveis de infligir risco à condição cardiovascular tende a manifestar-se, em síntese, por intermédio da SM, principalmente quando associadas às diversidades hormonais caracterizadas na disfunção ovulatória e no hiperandrogenismo. Os estudos sobre a associação da AN com a SM que tentam evidenciar essa conjunção cutâneo-metabólica ainda são precários. Nesse sentido, o presente estudo procurou estabelecer a prevalência da $\mathrm{AN}$ no contexto da SOP, além de suscitar as devidas implicações com a obesidade, a RI, a insulinemia basal e, em especial, a SM, dado que, por continuidade, exalta o risco cardiovascular dessas pacientes ${ }^{8}$.

\section{Métodos}

O estudo foi transversal e prospectivo. As pacientes foram relacionadas no Setor de Ginecologia Endócrina do Instituto de Ginecologia da Universidade Federal do Rio de Janeiro (UFRJ). Como critérios de inclusão, foram selecionadas cem pacientes portadoras de SOP (diagnosticadas segundo o critério de Rotterdam, 2003) $)^{9}$. No período compreendido entre maio de 2002 e dezembro de 2009, foram investigadas as inter-relações da AN com as testosteronas total e livre, a insulinemia basal, o estado de RI, a glicemia de jejum e os fatores de risco cardiovascular que se agrupam e ensejam a classificação da $\mathrm{SM}^{10}$. Tal critério se baseia na inclusão de diferentes componentes: $\mathrm{CA} \geq 80 \mathrm{~cm}$; triglicerídeos $\geq 150 \mathrm{mg} / \mathrm{dL} ; \mathrm{HDL}-\mathrm{C}<50 \mathrm{mg} / \mathrm{dL}$; pressão arterial $\geq 130 / 85$; glicemia de jejum $\geq 100 \mathrm{mg} / \mathrm{dL}$. O diagnóstico se confirma pela presença de, pelo menos, três caracteres alterados, inclusive diante da existência de diabete tipo 2. Cumpre ressaltar a realização rotineira da avaliação da glicemia pós-prandial (GPP-2h). Os valores da glicemia de jejum $\geq 126 \mathrm{mg} / \mathrm{dL}$ e da GPP-2h $\geq 200 \mathrm{mg} / \mathrm{dL}$ identificam o estado diabético. Condizente com o perfil dermatológico, a descrição do exame cutâneo, além da AN, abrangeu a presença do hirsutismo (índice $\geq 8$ ) ${ }^{11}$ e da acne ${ }^{12}$. Referente às irregularidades menstruais, a ausência de sangramento vaginal por período maior que 6 meses foi definida como amenorreia, e o intervalo menstrual além de 45 dias, como oligomenorreia. A ovulação irregular foi detectada entre o $20^{\circ}$ e $24^{\circ}$ dia do ciclo por meio da dosagem de progesterona $(<3 \mathrm{ng} / \mathrm{mL}$ ), após 2 ciclos consecutivos. Vigente a falta prolongada de menstruação, o espessamento endometrial igual ou inferior a $5 \mathrm{~mm}$ procedeu-se às dosagens hormonais.

Os critérios de exclusão dizem respeito a endocrinopatias e enfermidades afins, exequíveis de causar, direta ou indiretamente, distúrbios propícios à analogia ou vínculo com a SOP. A restrição se estende ao uso de hormônios decorridos 60 dias antes da seleção das pacientes. Determinação idêntica quando do emprego de medicações, a exemplo da sinvastatina cálcica e da metformina, substâncias, entre outras, que interferem com o metabolismo lipídico. Não se convencionou como princípio diagnóstico da SOP a relação hormônio luteinizante (LH)/hormônio folículo-estimulante (FSH) elevada. Foram excluídas, por igual, as pacientes com história de tratamento com antibióticos ( $>1$ semana) nos últimos seis meses, tabagismo, adictos de álcool e drogas em geral. O impedimento se cumpriu, também, decorrente de doença vascular (renal ou coronária). 
Os dados clínicos avaliados consistiram em idade $(\geq 16$ anos), peso $(\mathrm{kg})$, altura $(\mathrm{m})$, IMC, com base na relação $\mathrm{kg} / \mathrm{m}^{2}$, e a mensuração da CA (alterada quando $\geq 80 \mathrm{~cm}$ ) com fita métrica não elástica disposta entre a porção inferior da última costela e a borda superior da crista ilíaca, com a paciente em posição ereta, durante a expiração. Foram definidos os grupamentos ponderais de acordo com o IMC: normal $(18,5-24,9)$, sobrepeso (25-30) e obesidade (>30). A pressão arterial $(\mathrm{mmHg})$ foi mensurada no braço direito, com a paciente sentada, após cerca de dez minutos em repouso.

Há de se chamar a atenção para o fato de os meios propedêuticos serem antecedidos, como de hábito, pela anamnese circunstanciada. Mereceram atenção os comemorativos a partir do início da puberdade: ganho ponderal/ sobrepeso/obesidade, irregularidades menstruais, abortamento, diabete gestacional, doença hipertensiva específica da gravidez, ganho de peso materno e do recém-nascido (informes reservados à inserção de antecedentes clínicos no rol de investigações a propósito da SOP).

As estimativas hormonais e metabólicas foram realizadas entre o $3^{\circ}$ e o $7^{\circ}$ dia do ciclo menstrual, sequente à coleta de sangue (até as $9 \mathrm{~h}$ ) precedida de jejum (12 h). Nesse intervalo também foi efetuado o exame ultrassonográfico, pelo que a exploração privilegiou a densidade do estroma ovariano, ampliado e luzente (plano transversal) e a localização periférica ou disseminada dos folículos ${ }^{13}$. De modo a refletir a sensibilidade à insulina, foi utilizado o bomeostatic model assessment of insulin resistance (HOMA-IR) - modelo matemático de aferição da resistência insulínica - por intermédio do HOMA Calculator ${ }^{14}$, com ponto de corte $\geq 1,8^{15}$. As análises se cumpriram, além das dosagens de glicose e insulina, acrescidas do teste oral de tolerância à glicose (TOTG, $75 \mathrm{~g})^{16}$. A investigação acrescentou, ainda, os parâmetros hormonais, tais como FSH, LH, estradiol, 17-hidroxiprogesterona, prolactina, cortisol, hormônio estimulante da tireoide (TSH), de modo a excluir outras causas que se apresentam ao diagnóstico diferencial com a SOP, assim como a avaliação das testosteronas total e livre, a fim de concluir sobre o estado de hiperandrogenismo e a possível correlação com a AN.

Em relação aos hormônios, utilizou-se o processo de quimioluminescência ACS:180 (Ciba Corning Diagnostics, Massachusetts, EUA); a insulina foi medida por meio do radioimunoensaio com contador gama; quanto à bioquímica, foram utilizados os aparelhos Selectra-um (Bayer) ou Express-plus (Chiron Diagnóstica, Califórnia, EUA).

No que se refere aos procedimentos estatísticos, foram levados em consideração o valor bruto, a percentagem, a média e o desvio padrão, com os valores máximos e mínimos encontrados (para variáveis contínuas). Os dados descritivos valeram-se do teste do $\chi^{2}$ para variáveis categóricas. Em relação à diferença entre as médias de variáveis contínuas (para categorias distintas), foi utilizado o teste $t$ de Student. O critério adotado para o coeficiente de significância $(\mathrm{p}<0,05)$ corroborou a análise.

O protocolo obteve aprovação pela Comissão de Ética em Pesquisa do Instituto de Ginecologia da Universidade Federal do Rio de Janeiro (UFRJ). O Termo de Consentimento Livre e Esclarecido (TCLE) foi assentido por todas as pacientes, conforme resolução no 196/96 do Conselho Nacional de Saúde.

\section{Resultados}

A Tabela 1 expõe as médias e desvios padrão (variáveis contínuas) e frequências relativas e absolutas (variáveis categóricas) com relação aos dados clínicos, bioquímicos, cutâneos e a SM. A prevalência da AN, $53 \%$, concorreu com o hirsutismo $(72 \%)$ e a acne (49\%). Convém salientar a incidência em larga medida (>95\%) da AN, predominantemente no pescoço (face posterior e lateral), isoladamente ou em combinação com outras áreas. A SM alcançou a prevalência de $36 \%$. Fato incomum a AN sobrepujar a acne, mesmo em proporção reduzida, não obstante a presença marcante do hirsutismo, como de hábito a manifestação cutânea preponderante no decurso

Tabela 1. Dados clínicos, bioquímicos, cutâneos e perfil metabólico (síndrome metabólica) relacionados ao total de pacientes com síndrome do ovário policístico

\begin{tabular}{|c|c|c|}
\hline Variáveis & $\%$ & Média $\pm D P$ \\
\hline Idade & & $25,7 \pm 4,8$ \\
\hline IMC ( $\left(\mathrm{kg} / \mathrm{m}^{2}\right)$ & & $30,6 \pm 9,3$ \\
\hline $\mathrm{CA}(\mathrm{cm})$ & & $92,0 \pm 18,7$ \\
\hline \multicolumn{3}{|l|}{ Ciclo menstrual } \\
\hline Amenorreia & 63 & \\
\hline Oligomenorreia & 28 & \\
\hline Normal & 9 & \\
\hline \multicolumn{3}{|l|}{ Hisurtismo } \\
\hline Sim & 72 & \\
\hline Não & 28 & \\
\hline \multicolumn{3}{|l|}{ AN } \\
\hline Sim & 53 & \\
\hline Não & 47 & \\
\hline \multicolumn{3}{|l|}{ Acne } \\
\hline Sim & 49 & \\
\hline Não & 51 & \\
\hline \multicolumn{3}{|l|}{ SM } \\
\hline Sim & 36 & \\
\hline Não & 64 & \\
\hline Glicose $\mathrm{mg} / \mathrm{dL}$ & & $91,2 \pm 8,0$ \\
\hline Insulina $(\mu \mathrm{UI} / \mathrm{mL})$ & & $15,2 \pm 13,7$ \\
\hline Testosterona total (ng/dL) & & $62,5 \pm 42,8$ \\
\hline Testosterona livre (ng/dL) & & $5,1 \pm 10,2$ \\
\hline
\end{tabular}

IMC: índice de massa corpórea; CA: circunferência abdominal; AN: acantose nigricante; SM: síndrome metabólica. Verifica-se a percentagem, as médias e os desvios padrão (variáveis contínuas) e incidências relativas e absolutas (variáveis categóricas). 
da SOP, em tese tão somente superada no computo clínico pela oligomenorreia. Em contrapartida, merece destaque a maior incidência da amenorreia (63\%). Excetuados os valores médios normais da glicose $(<100 \mathrm{mg} / \mathrm{dL})$ e da testosterona total $(14,0-76 \mathrm{ng} / \mathrm{dL})$, cabe apontar para os níveis alterados do IMC e da CA, da testosterona livre e da insulina frente aos limites individuais de normalidade $-0,3-3,2 \mathrm{pg} / \mathrm{mL}$ e $\leq 12 \mu \mathrm{IU} / \mathrm{mL}$, respectivamente.

A Tabela 2 apresenta os valores médios e respectivos desvios padrão de variáveis contínuas, e frequências relativas e absolutas das variáveis categóricas, em separado para os grupos de pacientes em que se faz presente ou ausente a AN. No que tange à comparação, as idades não se diferençaram de modo significativo, do mesmo modo que as características do ciclo menstrual, a acne, a glicemia de jejum e as testosteronas total e livre. Ao contrário, a evidência de AN estabeleceu correlação significativa com o hirsutismo, o IMC, a insulina, o HOMA-IR e a SM. Apesar de 51\% das pacientes serem acometidas pelo estado de RI (HOMAIR) alterado, vale mencionar a incidência de intolerância à glicose não tanto expressiva (8\%) e, sobretudo, a ausência de diabetes mellitus tipo 2 .

Referente ao perfil dermatológico relacionado à SM (Tabela 3), a ênfase recai sobre a associação $(\mathrm{p}<0,01)$ entre a SM e a $\mathrm{AN}$, ao contrário do hirsutismo $(\mathrm{p}=0,4)$ e da acne $(p=0,9)$, bem como o dado significativo entre a SM e o HOMA-IR $(\mathrm{p}=0,01)$. A correlação significativa restrita à $\mathrm{AN}$ se deve ao vínculo previsível da dermatose com a RI e, por extensão, com os distúrbios cardiovasculares. Nesse particular, cabe registrar a associação expressiva entre a $\mathrm{SM}$ e a $\mathrm{AN}$ em $86,1 \%$ das pacientes.

Tabela 3. Perfil dermatológico relacionado à síndrome metabólica

\begin{tabular}{|c|c|c|c|c|c|}
\hline & & & \multirow{3}{*}{$d f$} & \multirow{3}{*}{$\begin{array}{c}t \text { de } \\
\text { Student } \\
\text { ou teste } \\
\text { do } \chi^{2}\end{array}$} & \multirow{3}{*}{$\begin{array}{c}\text { Valor } \\
\mathbf{p}\end{array}$} \\
\hline & Não (64) & $\operatorname{Sim}(36)$ & & & \\
\hline & \multicolumn{2}{|c|}{ n (\%) ou média DDP } & & & \\
\hline HOMA-IR & $1,5 \pm 1,4$ & $2,3 \pm 1,4$ & 54,3 & $-2,5$ & 0,012 \\
\hline Hirsutismo & & & 1 & 0,54 & 0,46 \\
\hline Sim & 68,8 & 77,8 & & & \\
\hline Não & 31,2 & 22,2 & & & \\
\hline Acne & & & 1 & 0,003 & 0,95 \\
\hline $\operatorname{Sim}$ & 50,0 & 47,2 & & & \\
\hline Não & 50,0 & 52,8 & & & \\
\hline AN & & & 1 & 22,7 & $<0,01$ \\
\hline Sim & 34,4 & 86,1 & & & \\
\hline Não & 65,6 & 13,9 & & & \\
\hline
\end{tabular}

SM: síndrome metabólica; DP: desvio padrão; df: graus de liberdade; HOMA-IR: homeostatic model assessment of insulin resistance; AN: acantose nigricante; p: coeficiente de significância.

Tabela 2. Dados descritivos para os grupos de pacientes com e sem acantose nigricante

\begin{tabular}{|c|c|c|c|c|c|}
\hline \multirow{2}{*}{ Variáveis } & \multicolumn{2}{|c|}{ AN } & \multirow{2}{*}{$d f$} & \multirow{2}{*}{$\begin{array}{l}t \text { de Student } \\
\text { ou teste do } \chi^{2}\end{array}$} & \multirow{2}{*}{ Valor $p$} \\
\hline & Não $(n=47)$ & $\operatorname{Sim}(n=53)$ & & & \\
\hline Idade (média $₫ D P$ ) & $25,2 \pm 4,5$ & $26,1 \pm 5,1$ & 98 & $-0,99$ & 0,3 \\
\hline Ciclo menstrual (\%) & & & 2 & 4,35 & 0,1 \\
\hline Amenorreia & 55,3 & 69,8 & & & \\
\hline Oligomenorreia & 29,8 & 26,4 & & & \\
\hline Normal & 14,9 & 3,8 & & & \\
\hline Sim & 59,6 & 83,0 & & & \\
\hline Não & 40,4 & 17,0 & & & \\
\hline Acne (\%) & & & 1 & 0,01 & 0,9 \\
\hline Não & 53,2 & 49,1 & & & \\
\hline Sim & 46,8 & 50,9 & & & \\
\hline Glicose (média $\pm D P$ ) & $90,0 \pm 7,2$ & $92,3 \pm 8,5$ & 97,9 & $-1,4$ & 0,1 \\
\hline Testosterona total (média $\pm D P$ ) & $56,7 \pm 4,1$ & $67,9 \pm 54,6$ & 68,3 & $-1,3$ & 0,1 \\
\hline Testosterona livre (média $\pm D P$ ) & $3,10 \pm 1,7$ & $7,19 \pm 14,3$ & 43,1 & $-1,8$ & 0,07 \\
\hline Insulina basal (média DPP) & $10,9 \pm 5,6$ & $19,1 \pm 17,4$ & 62,7 & $-3,2$ & $<0,01$ \\
\hline HOMA-IR (média DDP) & $1,39 \pm 0,7$ & $2,2 \pm 1,4$ & 79,2 & $-3,7$ & $<0,01$ \\
\hline
\end{tabular}

AN: acantose nigricante; df: graus de liberdade; DP: desvio padrão; SM: síndrome metabólica; IMC: índice de massa corpórea; HOMA-IR: homeostatic model assessment of insulin resistance (método da homeostase glicêmica); p: coeficiente de significância.

Utilizado o teste do $\chi^{2}$ para a relação com as variáveis categóricas. Quanto à diferença entre as médias de variáveis contínuas, foi utilizado o teste t de Student; adotou-se o valor $\mathrm{p}<0,05$ para estabelecer a significância estatística. 


\section{Discussão}

A SOP faz jus à denominação "the thief of womanhood" ("o ladrão da identidade feminina"), em razão de evidências estéticas depreciadoras, especialmente o hirsutismo, a acne vulgar e a alopecia andrógena ${ }^{17}$. Além disso, a inclusão da obesidade contribui para o desenlace do estresse psíquico-social, em virtude de comprometida a autoestima e debilitada a qualidade de vida. As implicações se estendem à medida que se acrescem as anormalidades metabólicas relacionadas à $\mathrm{AN}$, advindas da ação insulínica prejudicada, e a consequente hiperinsulinemia compensatória. Indeclinável, aliás, o fato de fatores endócrinos e metabólicos influenciarem-se reciprocamente, tanto que as perturbações referentes à homeostase desempenham interferência decisiva no agravamento das lesões que integram o perfil dermatológico da SOP. Todavia, o quadro é composto por anormalidades, por vezes ainda incipientes, a partir de uma "causa" supostamente mediada pela "cumplicidade" entre genes e estilo de vida.

A despeito de estudos prévios abordarem o vínculo entre os elevados níveis de androgênios e as dermopatias peculiares à SOP, os conhecimentos seguem, contudo, insuficientes, nos termos em que se revelam perante a associação com os parâmetros metabólicos ${ }^{18}$. Ainda que vinculada à obesidade e ao diabetes tipo 2 , a prevalência da AN observa certa disparidade, conforme diferentes resultados recentes. Entre todas as dermatopatias relacionadas à SOP, a prevalência da AN ocupa posição ínfima $(5,2 \%)^{18}$, ao contrário de estudos com porcentagens maiores, a exemplo de $64,6 \%{ }^{19}$, particularmente focalizados nas relações com o diabetes tipo $2^{20}$. A discrepância envolve fatores étnicos, geográficos e, notadamente, a coexistência de múltiplas condições de risco para o diabetes.

Artigos recentes enfatizam a potencialidade da SOP como fator de risco independente no que tange às doenças cardiovasculares, de maneira especial o diabetes tipo 2. Confere-se ênfase a SOP associada à presunção significativa de sobrevir ao diabetes e a dislipidemia não subordinadas ao $\mathrm{IMC}^{21}$. Reiteram o fato de as investigações mostrarem a persistência da sintomatologia agravante relacionada à síndrome de modo a exigir metódica pesquisa do diabetes, ainda que inalterada a glicemia de jejum. Inferiu-se, contudo, a irrelevância do hiperandrogenismo na composição de complicações de monta. Não obstante os efeitos deletérios da SOP possam declinar com o avanço da idade e, em consequência, atenuada a síndrome com a supressão da irregularidade menstrual e do hiperandrogenismo, a possibilidade de desenvolvimento de diabete e complicações decorrentes permanece $^{22,23}$. Na presente investigação, exemplifica-se o impacto da SM, passível de associar-se aos componentes clássicos da SOP, visto que a prevalência registrada $(36 \%)$ se compara às investigações prévias ${ }^{24,25}$.

A alta especificidade e o elevado valor de predição negativo conferem valiosa atribuição à $\mathrm{AN}$ em pressupor a ocorrência da $\mathrm{SM}^{10}$. Em contrapartida, a baixa sensibilidade inviabilizou a dermatose como procedimento de rastreio. Além do mais, salientam que a utilidade clínica da AN como marcador deve levar em conta a sua avaliação em áreas aparentes da pele, principalmente no que se refere à identificação de pacientes de alto risco para diabetes tipo 2. Em paralelo, conclui-se como fatores significativos na predição da SM, em pacientes com SOP, a idade avançada, a obesidade e a $\mathrm{AN}^{22}$.

Cabe assinalar, em razão dos resultados por agora analisados, a extraordinária prevalência da AN associada de modo significativo, em especial à SM (fato recíproco) e à condição ponderal aumentada, além de correspondências significativas com a RI (HOMA-IR), a elevação da insulina basal e o hirsutismo, dados que confirmam os pressupostos já assinalados no rol das experiências clínicas.

Em face do exposto, tem-se, com base na SOP, o fato de associar-se a AN com o quadro de disfunção metabólica. Fatores como obesidade, RI, hiperinsulinemia e SM, inter-relacionados, tornam preponderantes as implicações que se distinguem no espaço da Saúde Pública. Depreende-se, independentemente da faixa etária, a importância de proceder à inspeção rotineira (principalmente em áreas expostas - pescoço) da AN, sinal que integra o quadro fenotípico grave da SOP e prevê as intermediações que culminam no enlace com o amplo conceito de doença cardiovascular.

\section{Referências}

1. Stoddart ML, Blevins KS, Lee ET, Wang W, Blackett PR; Cherokee Diabetes Study. Association of acanthosis nigricans with hyperinsulinemia compared with other selected risk factors for type 2 diabetes in Cherokee Indians: the Cherokee Diabetes Study. Diabetes Care. 2002:25(6): 1009-14.

2. Torley D, Bellus GA, Munro CS. Genes, growth factors and acanthosis nigricans. Br J Dermatol. 2002;147(6):1096-101.
3. Araújo LM, Viveiros AM, Lopes RC, Viana AC, Fukui RT, Ursich M. Acanthosis nigricans em mulheres obesas de uma população miscigenada: um marcador de distúrbios metabólicos. An Bras Dermatol. 2002;77(5):537-43.

4. Mirmirani P, Carpenter DM. Skin disorders associated with obesity in children and adolescents: a population-based study. Pediatr Dermatol. 2014;31 (2): 183-90. 
5. Lee AT, Zane LT. Dermatologic manifestations of polycystic ovary syndrome. Am J Clin Dermatol. 2007;8(4):201-19.

6. Shivaprakash G, Basu A, Kamath A, Shivaprakash P, Adhikari P, Rathnakar Up, et al. Acanthosis nigricans PCOS patients and its relation with type 2 diabetes mellitus and body mass at a tertiary care hospital in Southern India. J Clin Diagn Res. 2013;7(2):317-9.

7. Wang $\mathrm{CH}$, Lin WD, Bau DT, Chou IC, Tsai CH, Tsai FJ. Appearance of acanthosis nigricans may precede obesity: an involvement of the insulin/IGF receptor signaling pathway. BioMedicine. 2013;3(2):82-7.

8. Dassanayake AS, Kasturiratne A, Niriella MA, Kalubovila U, Rajindrajith $S$, de Silva AP, et al. Prevalence of acanthosis nigricans in an urban population in Sri Lanka and its utility to detect metabolic syndrome. BMC Res Notes. 2011 ; 4:25.

9. Rotterdam ESHRE/ASRM-Sponsored PCOS Consensus Workshop Group. Revised 2003 consensus on diagnostic criteria and longterm health risks related to polycystic ovary syndrome. Fertil Steril. 2004;81(1): 19-25.

10. Grundy SM, Cleeman JI, Daniels SR, Donato KA, Eckel RH, Franklin BA, et al.; American Heart Association; National Heart, Lung, and Blood Institute. Diagnosis and management of the metabolic syndrome: an American Heart Association/National Heart, Lung, and Blood Institute Scientific Statement. Circulation. 2005; 1 12(17):2735-52.

11. Escobar-Morreale HF, Carmina E, Dewailly D, Gambineri A, Kelestimur F, Moghetti $P$, et al. Epidemiology, diagnosis and management of hirsutism: a consensus statement by the Androgen Excess and Polycystic Ovary Syndrome Society. Hum Reprod Update. 2012;18(2): 146-70.

12. Slayden SM, Moran C, Sams WM Jr, Boots LR, Azziz R. Hyperandrogenemia in patients presenting with acne. Fertil Steril. 2001;75(5):889-92.

13. Balen AH, Laven JS, Tan SL, Dewailly D. Ultrasound assessment of the polycystic ovary: international consensus definitions. Hum Reprod Update. 2003;9(6):505-14.

14. University of Oxford. The Oxford Centre for Diabetes, Endocrinology and Metabolism [Internet]. HOMA Calculator. 2004. [cited 2014 Jun 10]. Available from: https://www.dtu.ox.ac.uk/homacalculator/

15. Geloneze B, Vasques ACJ, Stabe CFC, Pareja JC, Rosado LEFPL, Queiroz EC, et al. HOMA 1-IR and HOMA2-IR indexes in identifying insulin resistance and metabolic syndrome: Brazilian
Metabolic Syndrome Study (BRAMS). Arq Bras Endocrinol Metab. 2009;53(2):281-7.

16. Sacks DB, Arnold M, Bakris GL, Bruns DE, Horvath $A R$, et al. National Academy of Clinical Biochemistry. Position statement executive summary: guidelines and recommendations for laboratory analysis in the diagnosis and management of diabetes mellitus. Diabetes Care. $2011 ; 34(6): 1419-23$.

17. Kitzinger C, Willmott J. 'The thief of womanhood': women's experience of polycystic ovarian syndrome. Soc Sci Med. 2002;54(3):349-61.

18. Ozdemir S, Ozdemir M, Görkemli H, Kiyici A, Bodur S. Specific dermatologic features of the polycystic ovary syndrome and its association with biochemical markers of the metabolic syndrome and hyperandrogenism. Acta Obstet Gynecol Scand. 2010;89(2):199-204.

19. Wijeyaratne CN, Seneviratne R de A, Dahanayake S, Kumarapeli V, Palipane E, Kuruppu N, et al. Phenotype and metabolic profile of South Asian women with polycystic ovary syndrome (PCOS): results of a large database from a specialist Endocrine Clinic. Hum Reprod. 2011 ;26(1):202-13.

20. Kong AS, Williams RL, Rhyne R, Urias-Sandoval V, Cardinali G, Weller NF, et al. PRIME Net Clinicians. Acanthosis nigricans: high prevalence and association with diabetes in a practice-based research network consortium--a PRImary care Multi-Ethnic network (PRIME Net) study. J Am Board Fam Med. 2010;23(4):476-85.

21. Wang ET, Calderon-Margalit R, Cedars MI, Daviglus ML, Merkin SS, Schreiner PJ, et al. Polycystic ovary syndrome and long-term diabetes and dyslipidemia risk. Obstet Gynecol. $2011 ; 117(1): 6-13$.

22. Norman RJ, Dewailly D, Legro RS, Hickey TE. Polycystic ovary syndrome. Lancet. 2007;370(9588):685-97.

23. Chang RJ. Defining risk in women with polycystic ovary syndrome: a need for constant vigilance. Obstet Gynecol. $2011 ; 117(1): 1-2$.

24. Ehrmann DA, Liljenquist DR, Kasza K, Azzis R, Legro RS, Ghazzi MN; PCOS/Troglitazone Study Group. Prevalence and predictors of the metabolic syndrome in women with polycystic ovary syndrome. J Clin Endocrinol Metab. 2006;91(1):48-53.

25. Dokras A, Bochner M, Hollinrake E, Markham S, VanVoorhis B, Jagasia $\mathrm{DH}$. Screening women with polycystic ovary syndrome for metabolic syndrome. Obstet Gynecol. 2005;106(1):131-7. 